\title{
Regionalentwicklung in Ostdeutschland - die humangeographische Perspektive
}

\author{
Rezension zu Sören Becker / Matthias Naumann (Hg.) (2020): Regionalentwicklung \\ in Ostdeutschland. Dynamiken, Perspektiven und der Beitrag der Humangeographie. \\ Heidelberg: Springer Spektrum.
}

\section{Annegret Haase}

Abb. 1 Titel des Buches (Springer Spektrum.)

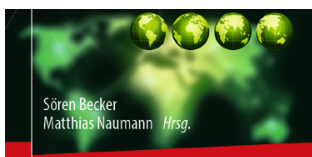

Regionalentwicklung in Ostdeutschland
Der von Sören Becker und Matthias Naumann herausgegebene Band bietet einen breiten Überblick über die Entwicklung und aktuelle Lage in Ostdeutschland aus einer raumbezogenen und rauminteressierten Perspektive. Er gibt an, vor allem den Beitrag der Humangeographie beizusteuern, jedoch zeigt der Blick auf das Kollektiv der beitragenden Autor_innen, dass der Blick doch weiter reicht als der der Humangeographie und stärker auch etwa ökonomische, demographische, soziologische, politikwissenschaftliche oder planerische Perspektiven einbezieht. Dieser erweiterte humangeographisch fokussierte Blick ist eine deutliche Referenz darauf, dass die Humangeographie heute zahlreiche Schnittmengen zu anderen disziplinären Sichtweisen aufweist - das kann man als Vorteil und Qualität einer großen Perspektivenvielfalt werten, oder aber auch als den Versuch, (fast) alle relevanten Themen in das Korsett der Humangeographie zu pressen. Hier mögen die Leser_innen selbst entscheiden, welche Sichtweise angemessener ist.

Der Band ist ein weiterer Versuch, eine Diagnose zum aktuellen Status quo sowie zu Entwicklungen in Ostdeutschland in den vergangenen 30 Jahren anzustellen. Er ordnet sich damit in eine immer weiter wachsende Landschaft von Publikationen zu diesem Thema in den vergangenen Jahren ein. Dennoch sticht er aus zwei Gründen hervor: Zum einen beleuchtet er beinahe enzyklopädisch eine Vielzahl verschiedener Themen und Bereiche, zum anderen wurde deren Auswahl durch die Kerninteressen der Humangeographie geleitet. Das Spektrum der Beiträge vereint - neben einer Einleitung - die sechs Bereiche Politische Geographie, wirtschaftlicher Strukturwandel, sozialer Wandel, Lage der Städte im Umbruch, Wandel des ländlichen Raumes sowie Mensch-Natur-Verhältnisse und Infrastrukturen. Auf diese verteilen sich nicht weniger als 38 Beiträge. Innerhalb dieser doch eher traditionell anmutenden Gliederung gibt es eine große Anzahl von Einzelbeiträgen zu sehr innovativen und neuen Themen, etwa zur postmigrantischen Überformung ostdeutscher Sozialwelten, neuen Abwehrallianzen an den deutschen Ostgrenzen, zum Thema der Rückwanderung (von West- nach Ostdeutschland) nach Jahrzehnten des Bevölkerungsexodus oder zu neuen Aspekten ländlicher Daseinsvorsorge. 
Die Auswahl der Beiträge spiegelt auch viele aktuelle Spannungsfelder und Herausforderungen Ostdeutschlands wider: Der Band geht ein auf das Thema der wachsenden Metropolen gegenüber schrumpfendem ländlichen Raum, auf die zunehmende Bedeutung von internationaler Zuwanderung und regressive Antworten wie Abwehr und Rassismus, auf Erfolg, Niedergang und Dauer-Wandel der ostdeutschen Wirtschaft, die Umgestaltung ganzer Regionen mit Blick auf den Kohleausstieg, die Polarisierung von politischen Einstellungen, den gefährdeten gesellschaftlichen Zusammenhalt sowie auf gesellschaftlichen Wandel als Dauerzustand und die resultierende Reaktion zwischen Anpassung, Abwehr und Protest.

In der Einleitung (Becker/Naumann 2020) sprechen die Herausgeber von Ostdeutschland als ,gewordener Region“(S. 1), welche sowohl geprägt ist durch die Zeit vor 1989 als auch die mittlerweile drei Jahrzehnte danach und die auch oft als Raumeinheit zur Beschreibung und/oder Analyse von Problemen oder aber Spezifika herangezogen wird. Daraus leiten sie auch ihre Begründung für die Herausgabe des Bandes ab: „Die fortdauernde Relevanz von Ostdeutschland - als komplizierte und differenzierte, aber doch weiterhin gerechtfertigte Raumeinheit - war für uns der Anlass für den Band ,Regionalentwicklung in Ostdeutschland“."(S. 2) Auf die reichhaltige Forschungsliteratur zum Thema wird kurz und fast nur aufzählend eingegangen, darauf folgt die genauere Einordnung des Bandes, der die bisherigen Perspektiven um eigene konzeptionelle und aktuelle empirische Befunde ergänzen möchte:

„Ziel des Bandes ,Regionalentwicklung in Ostdeutschland“ ist die Herausarbeitung der Widersprüche, der kleinräumlichen Ausdifferenzierung und der Konflikthaftigkeit räumlicher Entwicklung in Ostdeutschland seit der Wende. Mit dem regionalen Bezug auf Ostdeutschland nehmen wir in diesem Buchprojekt 30 Jahre nach dem Mauerfall zwar die Setzung vor, dass Ostdeutschland als räumliche Kategorie und Forschungsperspektive nach wie vor eine eigene Berechtigung hat. Wir möchten aber in konzeptioneller, empirischer wie auch disziplinärer Hinsicht den Blick erweitern, um die gängigen medialen wie auch wissenschaftlichen Zuschreibungen von ostdeutschen Städten und Regionen zu hinterfragen.“(S. 5)

Als Grundvoraussetzungen hierfür nennen sie: Ostdeutschland wird nicht als isolierte Raumeinheit aufgefasst, sondern der Fokus liegt auf kleinräumigen Unterschieden einerseits sowie der Ausleuchtung des Spannungsverhältnisses zwischen Differenzierung und Angleichung andererseits. Neben der räumlichen sei, so die Herausgeber, Ostdeutschland aber auch eine politische Region zwischen Postpolitik und „spaces of hope“ (Harvey 2000) im Sinne von Zukunftspotenzialen und Zukunftsfähigkeit. Entlang dieser Überlegungen sollen Fragen nach der Verallgemeinerung von Erkenntnissen über einzelne Teile Ostdeutschlands oder Herausforderungen für verschiedene Bereiche von Wirtschaft, Gesellschaft et cetera hinterfragt werden, wobei aber gleichzeitig durch die Natur des Bandes Verallgemeinerungen mit Bezug zu Ostdeutschland auch rekonstruiert und aufs Neue behauptet werden.

Einen Band von 500 Seiten in wenigen Worten auch in seinen Details $\mathrm{zu}$ würdigen beziehungsweise zu besprechen, ist ein sehr schwieriges 
Unterfangen. Daher habe ich im Folgenden einige Kapitel aus der Fülle der Beiträge für eine etwas detailliertere Betrachtung ausgewählt, die in ihrer Gesamtheit einen guten Überblick über aktuelle Debatten und/oder raumbezogene Herausforderungen im ostdeutschen Kontext geben sollen. Leitender Gedanke für ein selektives Vorgehen war, damit die Grundidee des Bandes, das Aufzeigen der Kompliziertheit und Komplexität, aber gleichzeitig eines inneren Entwicklungs- und Denkzusammenhangs der ostdeutschen Realität 30 Jahre nach der politischen Wende noch einmal nachzuvollziehen. Die Auswahl der Kapitel selbst muss letztlich ein Stück weit willkürlich und subjektiv bleiben, und sie sagt auch nichts über die Qualität oder Relevanz der berücksichtigten oder für diese Rezension beiseitegelassenen Themen aus - im Gegenteil soll trotz der Auswahl die ausdrückliche Anregung gegeben werden, sich von 500 Seiten nicht abschrecken zu lassen und das ganze Buch zu lesen!

Im Beitrag zu politischen Einstellungen (Kapitel 2: „Der ,Osten“ ist anders!? Anmerkungen zu den Diskursen über die politischen Einstellungen in Ostdeutschland“) thematisiert Manfred Rolfes blinde Flecken in der Debatte über das „Anderssein des Ostens“ (S. 19) und diskutiert, welche Bedeutung das Räumliche und „der Westen“ in der Konfiguration des „Ostens“ haben. Er verweist vor allem auf die Gefahr einer raumzentrierten Sichtweise und Erklärung:

„Solche räumlichen oder raumaffinen Plausibilisierungsversuche sind zwar bequem und erscheinen bisweilen naheliegend, sie erweisen sich aber immer wieder als unterkomplex und unangemessen. Sie verstellen - insbesondere wenn sie bewusst oder unbewusst postkolonial konnotiert sind - den Blick auf soziale, politische oder ökonomische Verursachungszusammenhänge.“ (S. 19)

Kristine Beurskens und Judith Miggelbrinks Beitrag zur Rolle der Bürgerwehren an der deutschen Ostgrenze wendet sich dem Phänomen des Vigilantismus zu, also des Handelns gegen unerwünschte Zustände oder Prozesse seitens nicht-staatlicher Akteure (Kapitel 6: „Geographien der Unsicherheit. Bürgerwehren an der ostdeutschen EU-Binnengrenze“). Die Autorinnen untersuchen die Bedeutung von Vigilantismus in Zeiten des Umbruchs sowie unter Bedingungen der Versicherheitlichung und Ent-Ortung, mit denen die Grenzregionen im Alltag konfrontiert sind. Im Ergebnis diagnostizieren die Autorinnen eine „grenzbezogene Kriminalitätsfurcht“ (S. 76) als Ursache der Entstehung von Bürgerwehren und diskutieren, ob die Verlagerung von Souveränität innerhalb der EU an den Grenzen auch als Schwäche des jeweiligen Staates wahrgenommen wird und damit Vigilantismus (indirekt) befördert.

In Kapitel 8 zum Thema „Kapitalmangel und Transferabhängigkeit. Zur politischen Ökonomie Ostdeutschlands" beleuchtet Dominik Intelmann die anhaltendeTransferabhängigkeit sowie das Fehlen einer lokalen Eigentümer_innenklasse als Herausforderungen sowie als Ursachen für die Schwäche der ostdeutschen Wirtschaft. Der Autor beleuchtet aus der Perspektive der politischen Ökonomie die langfristige Wirkung zentraler Entscheidungen der frühen Nachwendezeit wie die Unternehmensprivatisierung durch die Treuhandanstalt, die schnelle Währungsunion, die Altschuldenregelung und die Restitution (S. 101). 
In Kapitel 15 mit dem Titel „Ostdeutsche Identität im Wandel der Zeiten. 30 Jahre und noch kein Ende" wird die Frage der ostdeutschen Identität thematisiert, ein endloses (Streit-)Thema. Der Autor Daniel Kubiak schlussfolgert, dass es auch in der Nachwendegeneration eine kollektive Identifikation mit dem Osten gebe, welche sich aus den Erfahrungen der Umbruchzeit speise und darüber hinaus geprägt sei durch „[...] die Sozialisation über die Eltern, Schule und Medien, die Erfahrung symbolischer Abwertung als Ostdeutsche und die Ausgrenzung aus dem Normalitätsparadigma einer deutschen Identität“ (S. 189). Hier von einer Identität zu sprechen, welche eine ganze Generation charakterisiert, dem würde ich allerdings in dieser Ausschließlichkeit widersprechen. Und wie sind die vielen Westwanderer_innen einzuordnen?

Es ist erfreulich, dass das Thema Migration nicht vor allem durch die Themen Fremdenfeindlichkeit und Proteste gegen Fluchtzuwanderung abgehandelt wird. Die Migrationsgeschichte des Ostens seit 1989 wird sehr ausführlich in drei Beiträgen thematisiert, in zwei Kapiteln (17 und 18) zum Thema „Migrationsgeschichte Ostdeutschlands“ und in Kapitel 19, eher unerwartet, zum Thema „Ostdeutschland postmigrantisch und multikulturell“. Es wird zurecht als ein vernachlässigtes Thema angesprochen. Die Forderungen der Autor_innen um Jonathan Everts, diesem in der Forschung in Zukunft mehr Raum einzuräumen, unterstütze ich sehr. Durch meine eigene aktuelle Beschäftigung unter anderem mit migrationsbezogenen Konflikten in ostdeutschen Städten bin ich mir allerdings nicht sicher, ob die postmigrantische Perspektive tatsächlich die am besten geeignete ist, oder ob man nicht lieber von einer Migrationsgesellschaft sprechen sollte, wo Migration an einigen Orten (v. a. in einigen Großstädten) schon immer mehr zur Alltagsnormalität gehört, während das in vielen anderen Regionen (auch nach 2015/16) noch immer nicht der Fall ist.

Eine spezielle Perspektive aus dem großen Feld der Stadtentwicklung wird in Kapitel 27 mit dem Titel „Städtische Protestbewegungen in Leipzig. Orientierungsversuche innerhalb einer veränderten Marktrealität“ durch Rico Rokitte entworfen: Sein Blick richtet sich auf das urbane Protestgeschehen in Leipzig. Der Autor sieht die Geschichte dieser Proteste im engen Zusammenhang mit der Leipziger Nachwendeerfahrung massiver Schrumpfung (bis etwa 2000) und dynamischen Wachstums (seit 2010). Er stellt fest,

„dass die Leipziger Schrumpfungserfahrungen einen Schlüsselmoment für die Konstituierung, Artikulation und Positionsbestimmung städtischer Protestbewegungen darstellen. Dies betrifft einerseits die Perspektive der alternativen Subkulturen auf die Stadt selbst, andererseits deren Reaktion auf neoliberale Einhegungsstrategien.“ (S. 347)

Das Leipziger Beispiel wird als „Konfliktformation“ (S. 356) theoretisiert und aus der Perspektive der verschiedenen Formen der Forderung eines Rechts auf Stadt (mit Bezug zu Lefebvre) ausgeleuchtet. Wichtig scheinen mir zwei Dinge: Sowohl die Betrachtung der Leipziger Spezifik, welche die Stadt als eine der „Armen“ (S. 349) und der „Träumer“ (S. 350) beschreibt, die aus ganz unterschiedlichen Antrieben heraus und mit verschiedenen Zielen protestieren, als auch das generelle Anliegen, urbane Protestbewegungen 
im Kontext der neoliberalen Neuordnung der Städte genauer und kritisch zu analysieren, was der Autor stark einfordert.

Eine ganz andere ostdeutsche Erfahrung der vergangenen Jahrzehnte spricht dagegen Annett Steinführer im Kapitel zur Daseinsvorsorge im ländlichen Raum an (Kapitel 29: „Daseinsvorsorge in ländlichen Räumen: zwischen Abbau, Umbau und Ausbau“). Die Autorin wendet sich gegen die verbreitete These eines generellen Abbaus von Infrastruktur, welche für sie zu kurz greift (S. 384). Sie zeigt auf, dass es hier in manchen (vor allen technischen) Bereichen auch einen Ausbau sowie Umbau gab und dass der Kontext des Nachwende-Ostdeutschlands für ein Verstehen der aktuellen Herausforderungen für die ländliche Daseinsvorsorge nicht ausreicht, sondern dass hier auch übergreifende politische und ökonomische Rahmungen stärker zu berücksichtigen sind. Und sie spricht von nach wie vor bestehenden Wissenslücken trotz 30 Jahren postsozialistischer Regionalentwicklung (und auch Regionalforschung, S. 385).

Nicht zuletzt soll der Beitrag zur Sanierung ostdeutscher Industrieregionen von Gerd Lintz und Peter Wirth (Kapitel 36: „Sanierung alter Industrieregionen in Ostdeutschland“) hervorgehoben werden, vor allem, weil der gegenwärtige Prozess des Kohleausstiegs und der dadurch nötigen regionalen Umstrukturierung eine Dimension und Bedeutung erlangt hat, welche den gesamten politischen und gesellschaftlichen Diskurs zu Ostdeutschland wieder einmal voll entfacht hat - allerdings unter anderen Bedingungen als jenen der 1990er Jahre, wo ja bereits tatsächlich die meisten Arbeitsplätze im Kohlebergbau verschwanden. Der Beitrag stellt die Sanierung des Lausitzer Seenlandes nach der Braunkohle in den Mittelpunkt. Er stellt fest, dass die Sanierung auch international betrachtet durchaus eine Erfolgsgeschichte ist, und dass ohne sie auch die wirtschaftliche Entwicklung, wie sie sich trotz insgesamt noch nicht zufriedenstellender Ergebnisse bis heute vollzogen hat, nicht zustande gekommen wäre (478). Umweltsanierung und wirtschaftlicher Strukturwandel werden als großflächige Phänomene mit dem Umbau der Kohleregionen in Ostdeutschland erhalten bleiben.

In seiner Gänze bietet der Band eine Fülle von Material und spannenden Einzelbeiträgen; er richtet sich in erster Linie an raumbezogen arbeitende Wissenschaftler_innen und Studierende, ist aber auch gut lesbar für Interessierte außerhalb des wissenschaftlichen Spektrums. Für den Schluss nach 500 Seiten hätte man sich noch eine Systematisierung vonseiten der Herausgeber gewünscht, die einzelne Schlussfolgerungen aus den Kapiteln in einen näheren Bezug zueinander setzen. Hier müssen sich die Leser_innen selbst Gedanken machen. Schade ist das Fehlen eines solchen übergreifenden Beitrages am Ende vor allem für diejenigen, die nicht alles lesen wollen/ können und trotzdem an einem übergreifenden Blick, der über die anfängliche Einführung in die Beiträge hinausgeht, interessiert sind. Abschließend bleibt die Frage aber offen, inwieweit eine separate Beschäftigung mit Ostdeutschland in Zukunft weiter Sinn macht oder ob sie eventuell auch neue regionale Spezifika, Gemeinsamkeiten und Unterschiede zu wenig berücksichtigt, 30 Jahre nach der politischen Wende. Vielleicht wäre für die Zukunft auch eine humangeographische Perspektive auf die gesamtdeutsche Regionalentwicklung sinnvoll, die ostdeutsche mit anderen Spezifika sowie ältere und neuere regionale Unterschiede im Zusammenhang betrachtet. 


\section{Autor_innen}

Annegret Haase ist Stadtsoziologin und arbeitet u. a. zu nachhaltiger Stadtentwicklung, sozialräumlicher Differenzierung und Stadtumwelt-Themen.

annegret.haase@ufz.de

\section{Literatur}

Becker, Sören / Naumann, Matthias (Hg.) (2020): Regionalentwicklung in Ostdeutschland. Dynamiken, Perspektiven und der Beitrag der Humangeographie. Heidelberg: Springer Spektrum.

Becker, Sören / Naumann, Matthias (2020): Regionalentwicklung in Ostdeutschland Geographien einer Transformation. Zur Einleitung. In: Sören Becker / Matthias Naumann (Hg.), Regionalentwicklung in Ostdeutschland. Dynamiken, Perspektiven und der Beitrag der Humangeographie. Heidelberg: Springer Spektrum, 1-15.

Beurskens, Kristine / Miggelbrink, Judith (2020): Geographien der Unsicherheit. Bürgerwehren an der ostdeutschen EU-Binnengrenze. In: Sören Becker / Matthias Naumann (Hg.), Regionalentwicklung in Ostdeutschland. Dynamiken, Perspektiven und der Beitrag der Humangeographie. Heidelberg: Springer Spektrum, 71-83.

Everts, Jonathan / Juraschek, Kim Anna / Fleischmann, Larissa / Ringel, Florian (2020): Ostdeutschland multikulturell und postmigrantisch. In: Sören Becker / Matthias Naumann (Hg.), Regionalentwicklung in Ostdeutschland. Dynamiken, Perspektiven und der Beitrag der Humangeographie. Wiesbaden: Springer, 235-248.

Glorius, Birgit (2020): Migrationsgeschichte Ostdeutschlands. In: Sören Becker / Matthias Naumann (Hg.), Regionalentwicklung in Ostdeutschland. Dynamiken, Perspektiven und der Beitrag der Humangeographie. Heidelberg: Springer Spektrum, 211-222.

Harvey, David (2000). Spaces of hope. Edinburgh: Edinburgh University Press.

Intelmann, Dominik (2020): Kapitalmangel und Transferabhängigkeit. Zur politischen Ökonomie Ostdeutschlands. In: Sören Becker / Matthias Naumann (Hg.), Regionalentwicklung in Ostdeutschland. Dynamiken, Perspektiven und der Beitrag der Humangeographie. Heidelberg: Springer Spektrum, 99-110.

Kubiak, Daniel (2020): Ostdeutsche Identität im Wandel der Zeiten. 30 Jahre und noch kein Ende. In: Sören Becker / Matthias Naumann (Hg.), Regionalentwicklung in Ostdeutschland. Dynamiken, Perspektiven und der Beitrag der Humangeographie. Heidelberg: Springer Spektrum, 189-198.

Lintz, Gerd / Wirth, Peter (2020): Sanierung alter Industrieregionen in Ostdeutschland. In: Sören Becker / Matthias Naumann (Hg.), Regionalentwicklung in Ostdeutschland. Dynamiken, Perspektiven und der Beitrag der Humangeographie. Heidelberg: Springer Spektrum, 467-480.

Rokitte, Rico (2020): Städtische Protestbewegungen in Leipzig. Orientierungsversuche innerhalb einer veränderten Marktrealität. In: Sören Becker / Matthias Naumann (Hg.), Regionalentwicklung in Ostdeutschland. Dynamiken, Perspektiven und der Beitrag der Humangeographie. Heidelberg: Springer Spektrum, 347-359.

Rolfes, Manfred (2020): Der „Osten“ ist anders!? Anmerkungen zu den Diskursen über die politischen Einstellungen in Ostdeutschland. In: Sören Becker / Matthias Naumann (Hg.), Regionalentwicklung in Ostdeutschland. Dynamiken, Perspektiven und der Beitrag der Humangeographie. Heidelberg: Springer Spektrum, 19-30.

Steinführer, Annett (2020): Daseinsvorsorge in ländlichen Räumen: zwischen Abbau, Umbau und Ausbau. In: Sören Becker / Matthias Naumann (Hg.), Regionalentwicklung in Ostdeutschland. Dynamiken, Perspektiven und der Beitrag der Humangeographie. Heidelberg: Springer Spektrum, 375-388. 